\title{
The Comparison Study between Tuberculin Skin Test and Interferon Gamma Release Assay in BCG-Vaccinated Healthy Donors
}

\author{
Yoon-Sung Choi ${ }^{1,2}$ and Sunghyun Kim ${ }^{1,2, \uparrow}$ \\ ${ }^{I}$ Department of Clinical Laboratory Science, College of Health Sciences, \\ Catholic University of Pusan, Busan 46252, Korea \\ ${ }^{2}$ Clinical Trial Specialist Program for in vitro Diagnostics, Brain Busan 21 Plus Program, \\ Graduate School, Catholic University of Pusan, Busan 46252, Korea
}

\begin{abstract}
The incidence of tuberculosis (TB) in the Republic of Korea remains high when compared to the incidence in other Organization for Economic Cooperation and Development (OECD) countries. The prompt diagnosis and effective treatment of latent TB infection (LTBI) are very important in terms of controlling the burden of TB. The tuberculin skin test (TST) has long been the "gold standard" assay for the diagnosis of LTBI. However, it can show false positive results due to Bacille Calmette-Guérin (BCG) vaccination and infection with many environmental nontuberculous mycobacteria (NTM). The interferon gamma release assay (IGRA) using Mycobacterium tuberculosis (MTB)-specific antigens, was developed for the detection of LTBI. The QuantiFERON-TB Gold In-Tube assay is one of the most commonly used forms of the IGRA. In order to compare the diagnostic efficacy of the TST and IGRA in relation to LTBI among BCG-vaccinated healthy donors, whole blood samples were collected from 51 participants, and the results of the TST and IGRA were compared. Of the 51 cases, 18 cases (35.3\%) were positive and 33 cases (64.7\%) were negative when using the TST, while four cases $(7.8 \%)$ were positive and 47 cases $(92.2 \%)$ negative when using the IGRA. There was no correlation between the size of the induration in the TST and the IFN- $\gamma$ protein level. In conclusion, the TST showed higher crossreactivity among the BCG-vaccinated healthy participants, therefore, the IGRA might be the most suitable assay for the rapid screening of LTBI in BCG-vaccinated healthy population, or for TB contact investigation.
\end{abstract}

Key Words: Tuberculin skin test, Interferon gamma release assay, Latent tuberculosis infection, BCG vaccination, Contact investigation

Although the Republic of Korea has accomplished significant economic growth in recent decades and Koreans now generally enjoy a high standard of living, the country still has a high tuberculosis (TB) burden when compared to other Organization for Economic Cooperation and Development (OECD) member countries. Further, while the incidence of TB in the Republic of Korea declined from 100.8 per
100,000 people in 2011 to 76.8 per 100,000 people in 2016, the prevalence of latent TB infection (LTBI) among the elderly population is still estimated to be high (Kim and Yim, 2015; Kim and Kim, 2018).

Rapid diagnostic tests and the effective treatment of LTBI are very important in terms of reducing and controlling the burden of TB (Comstock et al., 1974; Hong et al., 2012). In

* Received: February 23, 2018 / Revised: May 30, 2018 / Accepted: June 1, 2018

${ }^{\dagger}$ Corresponding author: Sunghyun Kim. Department of Clinical Laboratory Science, College of Health Sciences, Catholic University of Pusan, Busan 46252, Korea.

Tel: +82-51-510-0560, Fax:+82-51-510-0568, e-mail: shkim0423@cup.ac.kr

(C) The Korean Society for Biomedical Laboratory Sciences. All rights reserved.

(C) This is an Open Access article distributed under the terms of the Creative Commons Attribution Non-Commercial License (http://creativecommons.org/licenses/by-nc/3.0/) which permits unrestricted non-commercial use, distribution, and reproduction in any medium, provided the original work is properly cited. 
particular, the accurate diagnosis of LTBI in individuals who face the highest risk of developing the active disease is essential (Recheldi, 2006). The tuberculin skin test (TST) has long been the "gold standard" assay for the diagnosis of LTBI (Pouchot et al., 1997; Kim et al., 2013). The TST detects a cutaneous delayed-type hypersensitivity response to the mycobacterial purified protein derivative (PPD), which contains over 200 Mycobacterium tuberculosis (MTB) proteins. Therefore, it shows cross-reactivity with the Bacille Calmette-Guérin (BCG) vaccination, as well as infection or contact with many environmental nontuberculous mycobacteria (NTM), since the PPD shares some major antigens with the BCG and several NTM (Comstock et al., 1974).

A recently introduced alternative immunodiagnostic method for the detection of LTBI is the interferon gamma (IFN- $\gamma$ ) release assay (IGRA) (Lalvani et al., 2001). The IGRA investigates the presence of MTB infection by detecting the ex vivo release of IFN- $\gamma$ by the immune cells following stimulation with MTB-specific antigens, for example, early secretory antigenic target 6 (ESAT-6) and culture filtrate protein 10 (CFP-10). These antigens are MTBspecific, since they are absent in all BCG strains and the major environmental NTM (Harboe et al., 1996; Mahairas et al., 1996; Shah et al., 2012), and they are also strong targets of the T-cells in MTB infection. The various available forms of IGRAs have shown significant potential to reduce falsepositive results, particularly among low-risk BCG-vaccinated populations. However, the IGRAs have also been known to exhibit only limited sensitivity for immunocompromised individuals and young children. Furthermore, the IGRAs cannot differentiate between the active TB disease and LTBI (Mandalakas et al., 2008; Kim et al., 2014).

Therefore, the present study aimed to compare the diagnostic efficacy of the TST and the IGRA in relation to the diagnosis of LTBI among BCG-vaccinated healthy donors. In order to accomplish this, whole blood samples from a total of 51 participants were collected, and the results of the TST and IGRA were compared.

From November 2010 to March 2013, a prospective clinical study was conducted. All participants provided written informed consent, and the study was approved by the Institutional Ethics Committee (approval number 4-2010-
0527). The healthy (non-TB) group was comprised of healthy adults who were free of active TB symptoms and who did not have any contact with active pulmonary TB patients. Potential participants in both the LTBI and healthy (nonTB) control groups were excluded if they showed clinical symptoms or demonstrated an abnormality on chest X-rays.

The TST was performed by means of the intradermal injection of two tuberculin units of purified protein derivative (PPD, RT23; Statens Serum Institute, Copenhagen, Denmark) according to the Mantoux method. The TST induration was measured following the injection for 48 to $72 \mathrm{~h}$. The cut-off value for positivity was $10 \mathrm{~mm}$.

The antigen stimulation of the whole blood cells was performed using commercial antigen tubes, namely the QFT-IT test (Cellestis Ltd., Carnegie, Australia), according to the manufacturer's instructions (Mori et al., 2004; Harada et al., 2008). The whole blood samples were collected into three QFT-IT collection tubes for the nil (negative control), MTBspecific antigen stimulation, and T-cell mitogen stimulation (positive control), respectively. The nil control tube contained only the anticoagulant, namely lithium heparin, which is used for detecting the basal level of immune response of peripheral blood cells. The MTB-specific antigen tube contained the anticoagulant and MTB-specific antigens, such as ESAT-6, CFP-10, and TB7.7, which are used for detecting the MTB-specific immune response. The T-cell mitogen control tube contained the anticoagulant and T-cell mitogen (phytohemagglutinin; PHA), which is used for detecting the immune cell function as a positive control. Antigen stimulation should be performed with in $3 \mathrm{~h}$ of the peripheral blood collection. In this study, antigen stimulation was performed for $24 \mathrm{~h}$ at $37^{\circ} \mathrm{C}$ using the QFT-IT test (IFN- $\gamma$ ELISA). Plasma was collected by means of centrifugation at $2,000 \times \mathrm{g}$ for $5 \mathrm{~min}$ and then stored at $-80^{\circ} \mathrm{C}$ until it was assayed for the QFT-IT test. The IFN- $\gamma$ concentration in the plasma samples obtained from each subject was determined using the QFT-IT ELISA test according to the procedure outlined in the product insert. The test results were interpreted using the QFT-IT ELISA software (version 2.43; Cellestis Ltd., Victoria, Australia), and the cut-offs for diagnosis outlined in the manufacturer's instructions were used. The mitogen stimulation served as an intrinsic control for 
Table 1. Comparison of IFN- $\gamma$ ELISA (QFT-IT) and tuberculin skin test (TST) results

\begin{tabular}{lccc}
\hline \hline & IFN- $\gamma$ ELISA positive & IFN- $\gamma$ ELISA negative & Total \\
\hline TST positive & 0 & 18 & 18 \\
TST negative & 4 & 29 & 33 \\
\hline Total & 4 & 47 & 51 \\
\hline
\end{tabular}

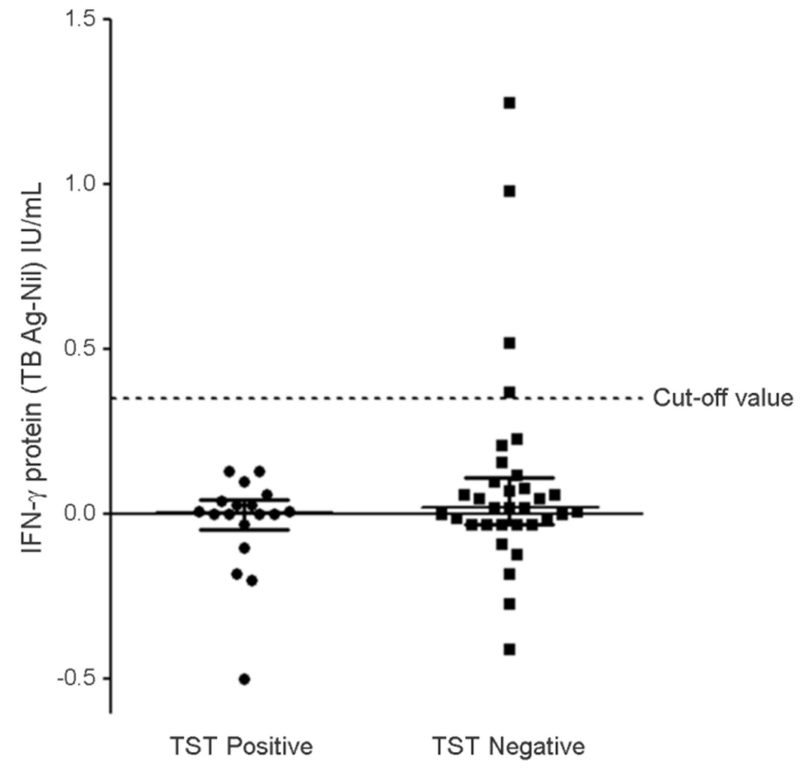

Fig. 1. Comparison of IFN- $\gamma$ protein level between TST positive and negative group of the TB intermediate burden country healthy donors after MTB specific antigens (ESAT-6, CFP-10, TB7.7) stimulation for $24 \mathbf{h}$. Whole blood of study subjects (TST positive and negative healthy donors) were treated with MTB specific antigens (ESAT-6, CFP-10, TB7.7) for $24 \mathrm{~h}$. The IFN- $\gamma$ protein level was analyzed by ELISA and the median value of TST negative group in healthy donor was slightly higher than TST positive group.

the blood sample quality. An IFN- $\gamma$ concentration of $\geq 0.35$ $\mathrm{IU} / \mathrm{mL}$ (following the subtraction of the IFN- $\gamma$ concentration of the nil control) after exposure to MTB antigens was considered positive for the QFT-IT, while a concentration of $<0.35 \mathrm{IU} / \mathrm{ml}$ was considered negative. If the IFN- $\gamma$ response to the mitogen was $>0.5 \mathrm{IU} / \mathrm{mL}$ higher than that of the nil control or $>8 \mathrm{IU} / \mathrm{mL}$ higher than that of the nil control, the result was deemed to be indeterminate (Mori et al., 2004).

Among the 51 samples, there were no double positives according to either the TST or IFN- $\gamma$ ELISA, and 29 samples yielded negative results in both examinations. Among the 18

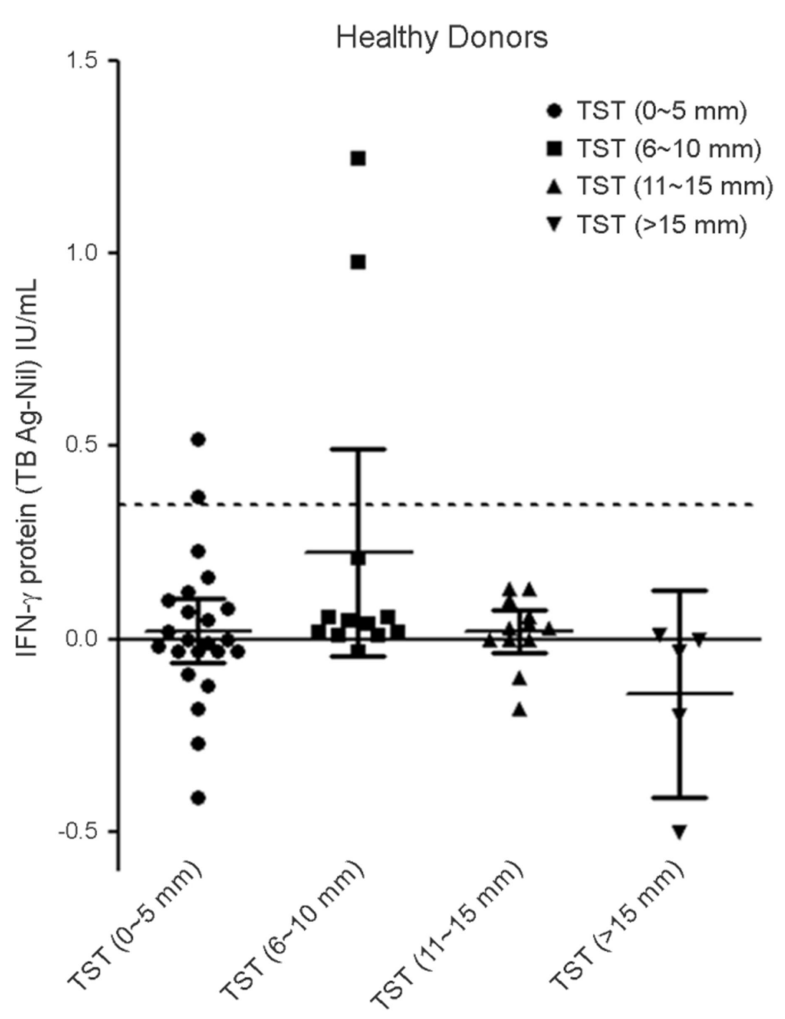

Fig. 2. IFN- $\gamma$ protein levels according to the tuberculin skin test (TST) results (sizes). In order to investigate the correlation between the size of the induration in the TST and IFN- $\gamma$ protein levels, healthy individuals were divided into four groups by the size of the induration $(0 \sim 5,6 \sim 10,11 \sim 15$, and $>15 \mathrm{~mm})$. The median value of IFN- $\gamma$ protein level was the highest in TST size $6 \sim 10 \mathrm{~mm}$ and the median value of IFN- $\gamma$ protein was the lowest in TST size over $15 \mathrm{~mm}$.

TST-positive samples, all the samples (18 samples) yielded negative results when using the IFN- $\gamma$ ELISA. Among the 33 TST-negative samples, four samples yielded positive results when using the IFN- $\gamma$ ELISA. These results indicate significant discordance between the TST and IFN- $\gamma$ ELISA results of healthy individuals (Table 1). There are currently only two commercialized LTBI diagnostic assays available, namely the TST and IGRA. Therefore, further analysis is 
required to evaluate the accuracy and reliability of those assays with alternative biomarkers such as TNF- $\alpha$, CXCL9, and CXCL11, which are highly specifically expressed in MTB-specific antigen-stimulated whole blood samples of active TB patients when compared to LTBI and normal participants (Kim et al., 2015).

In order to investigate the plasma IFN- $\gamma$ protein levels in response to the MTB-specific antigens (ESAT-6, CFP-10, and TB7.7) in healthy controls ( $24 \mathrm{~h}$ stimulation), the plasma IFN- $\gamma$ protein levels were detected using the IFN- $\gamma$ ELISA. Among the TST-negative samples obtained from the healthy control group, four samples yielded positive results when using the IFN- $\gamma$ ELISA (QFT-IT) (MTB antigen-nil $=>$ $0.35 \mathrm{IU} / \mathrm{mL}$ ). The median value of the IFN- $\gamma$ protein levels in the TST-negative samples was slightly higher than that in the TST-positive samples (Fig. 1), although the difference was not statistically significant.

Further, to investigate the correlation between the size of the induration in the TST and the IFN- $\gamma$ protein levels, the healthy individuals were divided into four groups according to the size of the induration $(0 \sim 5,6 \sim 10,11 \sim 15$, and $>15$ $\mathrm{mm})$. The median value of the IFN- $\gamma$ protein level in response to the MTB-specific antigens (ESAT-6, CFP-10, and TB7.7; $24 \mathrm{~h}$ stimulation) was the highest in the $6 \sim 11$ $\mathrm{mm}$ group, while it was the lowest in the $>15 \mathrm{~mm}$ group (Fig. 2). These results indicate that there was no correlation between the size of the induration in the TST and the IFN- $\gamma$ protein level in healthy donors' samples when the samples were stimulated with MTB-specific antigens.

In conclusion, the TST shows the higher cross-reactivity among BCG-vaccinated healthy participants, while the size of the induration in the TST does not exactly reveal the IFN- $\gamma$ protein levels in BCG-vaccinated healthy individuals. Therefore, the IGRA might be the most suitable assay for the rapid screening of LTBI in a BCG-vaccinated healthy population, or for prompt TB contact investigation. However, further investigation using more clinical samples should be undertaken in order to better determine the clinical usefulness of the two assays.

\section{ACKNOWLEDGMENTS}

This paper was supported by RESEARCH FUND from
Catholic University of Pusan.

\section{CONFLICT OF INTEREST}

The authors have no conflicts of interest to disclose.

\section{REFERENCES}

Comstock GW, Livesay VT, Woolpert SF. The prognosis of a positive tuberculin reaction in childhood and adolescence. Am J Epidemiol. 1974. 99: 131-138.

Harada N, Higuchi K, Yoshiyama T, Kawabe Y, Fujita A, Sasaki Y. Comparison of the sensitivity and specificity of two whole blood interferon-gamma assays for $M$. tuberculosis infection. J Infect. 2008. 56: 348-353.

Harboe M, Oettinger T, Wiker HG, Rosenkrands I, Andersen P. Evidence for occurrence of the ESAT-6 protein in Mycobacterium tuberculosis and virulent Mycobacteirum bovis and for its absence in Mycobacterium bovis BCG. Infect Immun. 1996. 64: 16-22.

Hong JY, Jung GS, Kim H, Kim YM, Lee HJ, Cho SN, Kim SK, Chang J, Kang YA. Efficacy of inducible protein 10 as a biomarker for the diagnosis of tuberculosis. Tuberculosis. 2012 e-publish.

Kim HW, Kim JS. Treatment of Latent Tuberculosis Infection and Its Clinical Efficacy. Tuberc Respir Dis. 2018. 81: 6-12.

Kim JH, Yim JJ. Achievements in and Challenges of Tuberculosis Control in South Korea. Emerg Infect Dis. 2015. 21: 1913 -1920 .

Kim S, Cho JE, Kim H, Lee D, Jeon B, Lee H, Cho SN, Kim YK, Lee H. Comparison of Mycobacterium tuberculosis Specific Antigen Stimulation Time for Performing Interferon Gamma mRNA Assay for Detecting Latent Tuberculosis Infection. J Exp Biomed Sci. 2013. 19: 90-97.

Kim S, Lee H, Kim H, Kim Y, Cho JE, Jin H, Kim DY, Ha SJ, Kang YA, Cho SN, Lee H. Diagnostic performance of a cytokine and IFN- $\boldsymbol{\gamma}$-induced chemokine mRNA assay after Mycobacterium tuberculosis-specific antigen stimulation in whole blood from infected individuals. J Mol Diagn. 2015. 17: 90-99.

Kim S, Park S, Lee H. Changes of Cytokine and Chemokine mRNA Expression in Whole Blood Cells from Active Pulmonary Tuberculosis Patients after T-Cell Mitogen and Mycobacterium tuberculosis Specific Antigen Stimulation. Biomedical Science Letters. 2014. 20: 162-167. 
Lalvani A, Pathan AA, McShane H. Rapid detection of Mycobacteirum tuberculosis infection by enumeration of antigenspecific T cells. Am J Respit Crit Care Med. 2001. 163: 824 $-828$.

Mahairas GG, Sabo PJ, Hickey MJ, Singh DC, Stover CK. Molecular analysis of genetic differences between Mycobacterium bovis BCG and virulent M. bovis. J Bacteriol. 1996. 178: 1274 -1282 .

Mandalakas AM, Hesseling AC, Chegou NN, Kirchner HL, Zhu X, Marais BJ. High level of discordant IGRA results in HIVinfected adults and children. Int J Tuberc Lung Dis. 2008. 177: 1164-1170.

Mori T, Sakatani M, Yamantigenishi F, Takashima T, Kawabe Y, Nantigenao K. Specific detection of tuberculosis infection: an interferon-gamma-based assay using new 430 antigens. Am J Respir Crit Care Med. 2004. 170: 59-64.

Pouchot J, Grasland A, Collet C, Coste J, Esdaile JM, Vinceneux
P. Reliability of tuberculin skin test measurement. Ann Intern Med. 1997. 126: 210-214.

Recheldi L. An update on the diantigennosis of tuberculosis infection. Am J Respir Crit Care Med. 2006. 174: 736-742.

Shah M, Dipietro D, Greenbaum A, Ketemepi S, Martins-Evora M, Marsiglia V, Dorman SE. Programmatic impact of QantiFERON-TB Gold In-Tube implementation on latent tuberculosis diagnosis and treatment in a public health clinic. PLoS One. 2012. 7: e36551.

https://doi.org/10.15616/BSL.2018.24.2.138

Cite this article as: Choi YS, Kim S. The Comparison Study between Tuberculin Skin Test and Interferon Gamma Release Assay in BCG-Vaccinated Healthy Donors. Biomedical Science Letters. 2018. 24: 138-142. 